جارعت دباط

Damietta University

Université de Damiette

Faculté de Pédagogie

Département de Curricula

et de Méthodologies

\title{
Utilisation des plateformes d'apprentissage électroniques pour développer les compétences professionnelles en français auprès des futurs enseignants
}

Recherche demandée pour la discussion de doctorat en Education( Didactique du FLE)

\section{Par}

\section{Mona Hossam ELdine Sapaa ELaich}

Chercheuse de la didactique de FLE

\section{Sous la direction de}

Prof.Dr.

Gamal El Chahat Abd ElHalim

Professeur-adjoint de curricula et

méthodologies de la langue

de française
Prof.Dr.

Mahmoud Ezz El-Arab Abd El Kade

Professeur-adjoint de curricula et

de méthodologies de la langue 


\section{Sommaire}

La recherche actuelle a pour but de vérifier l'effet d'une plateforme d'apprentissage électronique sur le développement des compétences professionnelles auprès des futurs enseignants. la formation initiale n'est pas seulement une question d'appropriation de savoirs. Il faut que les capacités operationnelles croissent et permettent aux futurs enseignants de maitriser de mieux en mieux le champ pédagogique. nous avons utilisé la méthode descriptive et analytique en exposant les concepts, les études antérieurs portant sur les compétences professionnelles et les plateformes électroniques d'apprentissage au service les compétences professionnelles. Nous avons aussi utilisé la méthode expérimentale en appliquant les outils de la recherche pour vérifier leurs impacts sur le développement des compétences professionnelles auprès l'échantillon ciblé. Nous avons conçu une plateforme (Microsoft Teams), et un pré/post test. Notre échantillon se compose de 30 étudiantes de troisième année, section de français, à la faculté de pédagogie,université de Damiette comme groupe expérimental. Les résultats de cette recherche ont montré que la plateforme électronique proposée a eu un impact significatif sur le développement des compétences professionnelles en FLE pour l'échantillon ciblé.

Mots-clés : plateformes électronique, compétences professionnelles- la plateforme Microsoft Teams. 


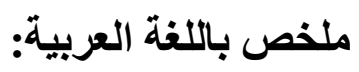

يهدف البحث الحالى إلى التحقق من أثر منصة تعلم إلكترونية على تتمية المهارات المهنية لدى الطلاب المعلمين .فالتكوين المبدئى

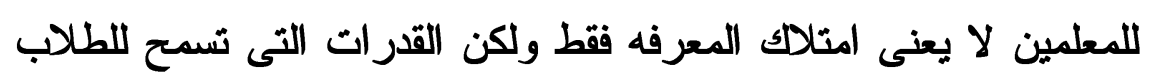
المعلمين بالتدريس بشكل أفضل فى الحقل التربوى .اعتمد البحث المثل المنهج

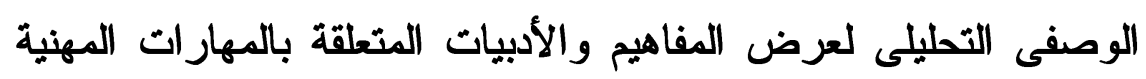

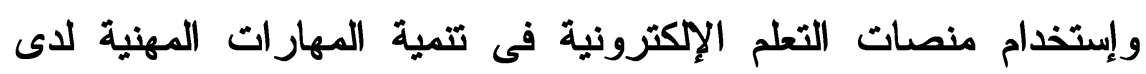

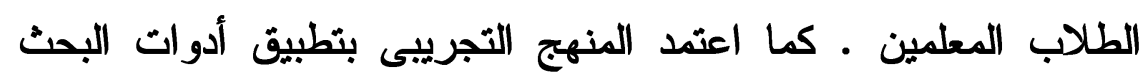

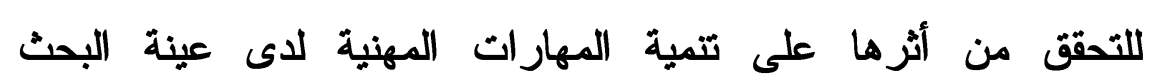

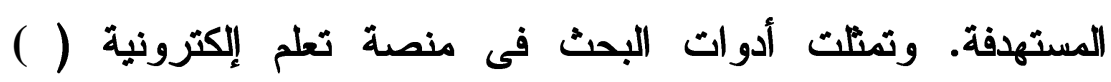
Microsoft Teams (30) طالبة من طلاب الفرقة الثالثة بكلية التربية, جامعة دمياط, كمجموعة تجريبية. وقد أسفرت نتائج البحث عن وجود أثر دال للمنصة الاكترونية المقترحة لتنمية المهارات المهنية لدى الطلاب المعلمين عينة البحث.

الكلمات المفتاحية: منصة التعلم الإكترونية ـ المهارات المهنيةــ منصة

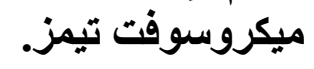




\section{1- Introduction :}

l'intégration de la technologie dans la classe nécessite une maîtrise de ces technologies, que ce soit pour enseigner ou accompagner les élèves dans leurs usages du numérique.

Les processus de développement professionnel sont considérés comme des programmes de renforcement cognitif pour les enseignants de français à la lumière des changements dans les théories d'apprentissage, les stratégies d'enseignement, les activités des enseignants et les nouveaux rôles. Avec la formation du professeur sur les compétences liées au développement de sa construction cognitive.

la plateforme "Microsoft Teams" est un réseau social conçu spécifiquement pour les participants dans le processus éducatif: enseignants, élèves et leur parents. peut être considéré comme une nouvelle façon d'utiliser les technologies pour l'apprentissage.

Plusieurs caractéristiques sont attribuées à une plateforme qui privilégie la construction des connaissances soit d'une manière individuelle ou collective. Ou crée un dynamisme et du lien social qui implique de la part des apprenants d'apprendre les compétences nécessaires au travail en équipe. Elle repose sur l'initiative individuelle et collective pour faire émerger le plus grand nombre d'initiatives permettant de valoriser l'apprenissage à distance. La plateforme agit au niveau collectif, elle permet de mieux connaître les apprenants afin d'initier des démarches collaboratives autour de projets concrets. 
Il permet aux enseignants de partager du contenu, de distribuer des questionnaires, des exércices et de gérer la communication avec les élèves et leur parents. Permettre d'apprendre à l'aide de différent matériel didactique:site web ou d'autres sites en ligne, livres ou autres textes, documents visuels ou sonores, vidéo, etc. et de communiquer via différents moyens de communication :courriel forum, message texte, visioconférence, etc.

les compétences professionnelles constituent une base essentielle pour tout enseignant de la langue française, surtout au début de son travail en classe. Parmi des chercheurs ont déclaré que la formation des enseignants a été trop théorique et pas assez pratique (Seyam, 2017;Ahmed,2018; Ezz El-arab, 2019)

\section{2 - Position de la problématique :}

Il est évident que les nouvelles technologies jouent un rôle important dans le processus d'enseignement et d'apprentissage offriraient des méthodes et moyens plus attrayantes pour l'apprenant, peut aider a mieux apprender, intéragir. Et les chercheurs ont mis en évidence l'importance de l'utilisation l'environement d'apprentissage électronique, et plus précisement la plateforme électronique dans l'enseignement et l'apprentissage des langues (AL Adl,2019 ; Elseify 2019, El-Fakharany, 2010, Abdalla,2020) ces études ont également recommandé de développer les compétences professionnelles et linguistiques afin que le futur enseignant puisse s'entraîner à une communication 
proche de la réalité où il ne maîtrise pas la compétence à gérer sa classe aura aussi des difficultés dans les autres facettes de son rôle, telles que celles de motivateur, de facilitateur, de négociateur ou d'organisateur.

En outre, pour confirmer les résultats des études antérieures, on a appliqué une étude explorative non standardisée à un échantillon des étudiants de 3ème année section de français à la faculté de pédagogie de Damiette pour l'année universitaire 2020-2021. Cette étude est une grille d'observation de quelques performances professionnelles nécessaires aux futurs enseignants $\mathrm{du}$ français pendant le processus d'enseignement. Les résultats de cette étude ont montré une faiblesse tangible dans les compétences professionnelles chez eux à cause de leur préparations et formation pédagogique insuffisantes.

\section{3 - Problématique de la recherche:}

Les résultats des études antérieures et étude explorative ont montré le besoin urgent du développement des compétences professionnelles. D'où vient l'importance d'éffectuer la présente étude.On peut formuler cette problématique dans la question principale suivante: "Quelle est l'efficacité d'émployer la plateforme $d$ 'apprentissage électronique pour développer les compétences professionnelles auprès des futurs enseignants?

Par conséquent, il est nécessaire de répondre aux questions secondaires suivantes ; 
1) Quelles sont les compétences professionnelles nécessaires pour les futurs enseignants en français?

2) $\mathrm{Au}$ quelle point les futurs enseignants de la section de français, à la faculté de pédagogie possedent-ils ces comptétnces professionnelles de la section de français, à la faculté de pédagogie?

3) Quelle est la représentation proposée pour utiliser la plateforme"Microsoft Teams" afin de développer les compétences professionnelles auprès les futurs enseignants de la section de français, à la faculté de pédagogie?

4) Quel est le degré d'efficacité de plateforme" Microsoft Teams" afin de développer les compétences professionnelles auprès les futurs enseignants de la section de français, à la faculté de pédagogie?

\section{4 - Objectifs de la recherche:}

Cette recherche vise essentiellement à atteindre les objectifs suivants :

1. La recherche peut contribuer à ouvrir de nouveaux domaines à d'autres chercheurs portant sur l'efficacité de l'utilisation de la plateforme électronique d'apprentissage «Microsoft Teams» pour développer les autres compétences langagières et d'enseignement. 
2. Développer les aptitudes des compétences professionnelles nécessaires pour les futurs enseignants de la section de français à la faculté de pédagogie de Damiette.

\section{5 - Importance de la recherche:}

\section{La recherche actuelle peut bénéficier les catégories suivantes:}

1) Présenter la manière de structure ce nouveau savoir, les compétences professionnelles pour le transmettre ou le faire acquérir au futur enseignant et en même temps adjuster le savoir a ses priorités et à ses exigences.

2) Fournir aux étudiants du département de français à la faculté de pédagogie les compétences professionnelles et développer leur capacité à les utiliser et à les actualiser.

3) Proposer une stratégie qu'il pourrait essayer à l'enseignement des compétences professionnelles futurs enseignants de section du français aux différents niveaux. à travers la plateforme éducative "Microsoft Teams" pour développer les compétences professionnelles.

4) Mettre à la disposition des auteurs du programme de français à la faculté de pédagogie les outils (outputs) de cette recherche,ce que pourrait les aider à construire le cursus de formation des futurs enseignants. 


\section{6- Délimites de la recherche:}

La recherche actuelle se limite à :

1) Un échantillon d'étudiants de la troisième année du département de français à la faculté de pédagogie de Damiette.

2) Certaines compétence professionnelle nécessaires pour les futurs enseignants de la troisième année du département de français à la faculté de pédagogie de Damiette.

3) . L'utilisation de la plateforme d'apprentissage électronique ( Microsoft Teams).

\section{7 - Matèriels et instruments de la recherche:}

La chercheuse a élabore les instruments suivants:

1. Un test pour mesurer le degré de développement les compétence professionnelle chez les étudiants de l'échantillon.

2. Programme basé sur des unités proposées pour employer la plateforme « MicrosoftTeams »afin de développer des compétences professionnelles.

\section{8- Hypothèses de la recherche:}

Cette recherche a essayé de mettre en preuve les hypothèses suivantes:

1. Il y a une différence significative statistiquement au niveau $(\leq 0,05)$ entre la moyenne des notes des étudiants de l'échantillon au pré/post test 
des compétences professionnelle en faveur du post test.

2. La plateforme électronique proposée a une taille d'éfficacité sur le développement des compétences professionnelles en FLE pour l'échantillon de la recherche.

\section{9-Procédures de la recherche:}

Pour répondre aux questions de l'étude, la chercheuse a suivi les démarches suivantes:

1) Consulter les littératures et les études précédentes arabes et étrangères se rapportent au sujet de la recherche actuelle et utiliser pendant la préparation de l'étude théorique et expérimentale.

2) Elaboration d'un test des compétences professionnelles

Standardiser les outils de la recherche (calcule de : validité, fidélité ,facteurs de facilité et de difficulté) et faire les modifications nécessaires.

3- Choisir l'échantillon de l'étude parmi les futurs enseignants de la troisième année du département de français à la faculté de pédagogie de Damiette .

4- Préparer un programme basé sur les plateformes électroniques en suivant les étapes suivantes ;

a) Déterminer les objectifs généraux et spécifiques du programme.

b) Préciser les differences sources du programme .

c) Préparer le contenu du programme . 
5-Présenter le programme au jury et le modifier dans sa forme finale .

6- Préparer le pré/ post test de compétences professionnelle, le présenter au jury et faire les ajustement nécessaires, et un traitement statistique.

7-Appliquer le test compétences professionnelle aux étudiants de l'étude.

8-Enseigner aux étudiants du groupe en utilisant le programme.

9- Ré-appliquer le test compétences professionnelle aux étudiants de groupe.

10- Analyser et interpréter les résultats statistiquement.

11 - Formuler des recommandations à la lumière des résultats de la recherché en cours.

\section{0- Terminologie de la recherche:}

\section{La plateforme électronique "Microsoft}

\section{Teams":}

Selon (El-Nasser,2013) est une plateforme de la communication sociale où le professeur peut discuter leurs grades, leurs tests, leurs devoirs aussi elle rassemble entre la plateforme "Facebook" et "Blackbord". On utilise la technologie Web 2.0. lorsque le professeur contrôle la plateforme en communiquant avec les étudiants à l'espace ouvert qui envoie et reçoit les messages textes et vocaux. 
Une plate-forme est un logiciel qui assiste la conduite des enseignements à distance. Ce type de logiciel regroupe les outils nécessaires aux principaux utilisateurs d'un dispositif qui a pour finalités la consultation à distance de contenus pédagogiques, l'individualisation de l'apprentissage et le télétutorat. (Ecoutin \& Even 2001 : 2) .

La chercheuse la définit comme un outil pédagogique qui permet de gérer et de donner accès à un ensemble d'activités et de ressources pédagogiques d'une manière synchrone ou asynchrone n'importe quel lieu et n'importe quel moment.

\section{Les compétences professionnelles :}

Selon

(Tardif,2006:22)

définit

la compétences"comme un savoir-agir complexe prenant appui sur la mobilisation et la combinaison efficaces d'une variété de ressources internes et externs à l'intérieur de situation d'apprentissage.

D'après (Elseify,2019:107) la compétence professionnelle est multidimensionnel. $\mathrm{Ce}$ terme exprime les connaissances de l'enseignant, ses convictions, ses ressources, ses hbilités et ses perspectives et ses tendances pour traiter efficacement la situation d'apprentissage en une performance réussie.

La chercheuse voit que les compétences professionnelles comme un ensemble de connaissances, de concepts, savoirs professionnels et attitudes que le futur enseignant doit perfectionner. 
Elle se réalise a travers deux volets dont l'un est pour acquérir le savoir -faire pendant le stage pratique"

\section{L'étude théorique :}

L'apprentissage virtuelle est un moyen de communiquer des connaissances, de communiquer, d'obtenir des informations et des formations via Internet, Parmi ces moyens les plateformes életronique qui sont utilisés pour mettre en ligne des cours, ce que permettre aux apprenants d'intéger l'usage des TIC dans leurs pratiques d'apprentissage et entraîner à entrer en contact avec les autres ou natifs francophones.

La formation sur l'usage de plateforme semblent être les facteurs favorisant l'intégration de la plateforme dans leurs pratiques d'enseignement. Le contact de l'enseignant avec d'autres personnes et ses interactions avec divers objets communicationnels Durant la formation, lui permettent de puiser des informations et des ressources dans ce dispositif technique pour accomplir les gestes d'appropriation pour reprendre l'expression de (Proulx et SaintCharles,2004:82)

\section{Les plateformes électroniques:}

Selon (Komis et al.2013) les plateformes construisent une avancée technologique qui amplifie les possibilités offertes par les TIC dans les différents contextes de formation universitaires ou secondaires. Cette avancée permet d'enseigner et d'apprendre en s'affranchissant des barrières physique, mais aussi 
pédagogiques et psychologiques qui contribuent au cloisonnement des forms traditionnelles d'enseignement(Abdalla,2020 : 115) .

On peut dire que les plateformes électroniques pour la pédagogie amène à faciliter l'accès des étudiants à de nombreuses ressources didactiques spécifiques ou sélectionnées sur Internet et d'envisager autrement les interactions entre les é tudiants et les enseignants et son susceptibles de les favoriser,

\section{Que veut dire la plateforme LMS?}

Les plateformes d'apprentissage sont habituellement appelées VLE (environnement d'apprentissage virtuel), Il existe environ plus de 200 plateformes d'apprentissage en ligne dont une trentaine sous licences libres. La norme SCORM(sharable Content Object Refernce Model) permet de transposer du contenu d'une plateforme à une autre plateforme e-learning.

Les plateformes de formation à distance sont des outils logiciels dont le rôle est de permettre le pilotage des enseignements à distance Le vocabulaire informatique utilise de façon conventionnelle le terme Learning Management System (LMS). On peut classer les LMS, selon leur architecture de base, selon leur mode de distribution (open source ou propriétaire), selon le langage informatique qu'ils utilisent, selon la philosophie de l'apprentissage qui a structuré leur construction, selon les types de marchés pour lesquels ils sont destinés (école, entreprise), etc. On trouve ainsi des LMS à orientation contenu vs compétence, des 
LMS à orientation autonomie vs collaboration et des LMS à orientation individualisation vs massification.

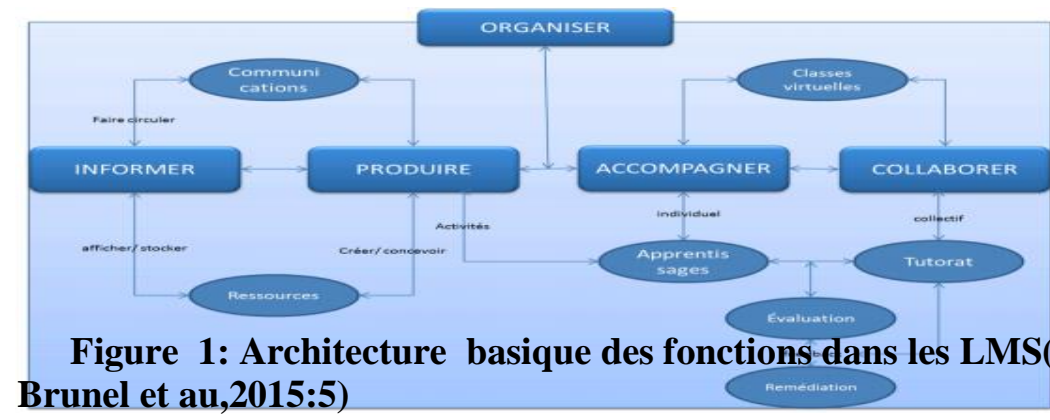

Des recherches montrent 1 'efficacité des plateformes dans le processus d'apprentissage et soulignent à elle qu' un outil efficace pour évaluer le travail des apprenants complexes. Des études ont également souligné l'importance de l'utilisation de ces plateformes telles que ,Et l'étude de Elseify (2019): qui ont utilisé les plateformes Aider les enseignants pour développer les méthodes d'enseignement traditionnelles à travers l'intégration d'une des applications technologiques, appelés par plateformes dans le processus d'enseignement et d'apprentissage et son impact sur l'apprentissage des étudiants .

Parmi les études qui collectionnent entre la plateforme et numériques et les competences, l'étude d'(AlAdl, 2019), qui s ' intérèsse à vérifier l'effet d'une plateforme numérique d'apprentissage sur le développement de la compétence de compréhension orale en FLE.. Et soulèvent des questions importantes 
liées à l'utilisation de la technologie dans l'apprentissage des langues étrangères l'évaluation et la distinction entre les niveaux d'une performance et se confirme sur le lien entre la téchnologie de l'information et de la communication (TIC) comme un environnement riche pour développer les processus d'apprentissage de langue. étude de (Hoda Elgamdy, 2019)

Qui a recommandé d'utilisation de plates-formes éducatives dans l'enseignement des mathématiques et d'autres matériels et l'utilisation de l'apprentissage en ligne à différents étape de l'enseignement general.

Les causes de la faiblesse des compétences professionnelles chez les futurs enseignants du FLE

\section{1-Manque d'emploi de TIC:}

Selon( Radi, 2001:23) voit que le déclin de motivation chez les étudiants et les chercheurs en Egypte . Actuellement, le fonctionnement de l'université est très critiqué en Egypte, tant en ce qui concerne l'infrastructure que l'enseignement. Les enseignants représentent la principale et parforis la seule source d'apprentissage pour les étudiants, le problème de matériel est bien sûr centrale dans la formation a la recherche et dans la pratique de cette dernière .

D'autre part; Plusieurs auteurs, comme affirment l'intérêt et les nombreux avantages, dans le contexte universitaire, du blogue, présenté comme cahier de laboratoire virtuel, inséré dans une plateforme elearning, le blogue permet d'offrir un apprentissage à 
distance. Cet outil est une brique pédagogique. Il en permet de mettre en forme le texte ds billets et gère les commentaires associés.

Cependant les études antérieures comme (Othman, 2008; Salem,2013; El seify, 2019, Al Adl,2019) ont visé à développer les compétences de l'écrit(lecture/écriture) et de l'écoute chez les étudiants universitaires- les futurs enseignants à partir de l'intégration des TIC dans le processus de l'enseignement enseignement. Il ya un manque dans les études égyptiennes menées sur ce domaine en FLE.

Pour ce la, il fallait la formation des futurs enseignants en Egypte s'intérer à 1)développement des connaissances de base tant à l'usage des TIC qu'a leur intégration dans le processus éducatif 2)développement des connaissances et des compétences appropriées pour la planification et la production des activités éducatives et des plans de cours en utilisant des TIC.

Par exemple quand ils utilisent les plateformes élèctroniques dans l'enseignement pour pouvoir présenter un cours en intégrant les TIC, il faut un ensemble de compétences et de connaissances dans ce cas, former les enseignants à l'usage des TIC c'est tout simplement leur favoriser un environement d'apprentissage.

\section{2-Manque d'intérêt au stage pratique:}

Beaucoup d'études antérieures ont montré que la période du stage pratique ne permet pas aux étudiants 
futurs-enseignants d'acquérir les compétences nécessaires à l'enseignement comme intégrer la TIC dans la classe et la gestion de la classe, et la planification de cours comme l'étude de (Abd Alrahman,2000; Abbas,2016; Seyam,2017 Ahmed,2018; Ezz El-arab, 2019)

Selon (Develay, 1994) souligne que pour la formation des enseignants, les savoirs théoriques ne prennent une réelle signification que s'ils donnent naissance à des pratiques, inversement, une pratique ne prend toute sa signification qu'elle est analysable avec les savoirs théoriques.

Comme l'affirme (Abd Alrahman,2000) nous devons accorder une grande importance au stage pratique qui est une période nécessaire pour aider l'étudiant futur enseignant à pratiquer et à maîtriser les compétences didactiques dans une atmosphère réelle et dans des situations vivantes. Et le stage pratique n'atteint pas ses objectifs espérés à cause de l'absence de l'entraînement de l'étudiant pendant le cours de méthodologie du FLE aux nouvelles techniques liées aux compétences de son enseignement.

Aussi (Gohar, 2015) indiquent que les méthodes de formation doivent refleter fidelement que possible l'exercice reel de l'enseignement (apprentissage contextuel). L'evaluation demontre le cheminement du developpement de la competence en impliquant les futurs enseignants de facon a ce qu'ils s'evaluaent

Dans le même orders des idées l'expertise pratique consisterait à faire face de manière satisfaisante a 
l'ensemble des situations d'enseignement et d'apprentissage. Cela implique aussi que le futur enseignant doit pouvoir résoudre des tâches complexes dans un contexte social singulier et pluriel. Il acquiert par conséquent son expertise pratique dans l'action à partir de sa manière d'être et en tenant compte de la micro société dans laquelle il évolue.

Malgré l'évolution de la conception de l'évaluation et les innovations qui concernent le changement des pratiques dans les classes se heurtent bien à des résistances. Comme les études (Osman,2018) et (Ahmed,2018) (Ibrahim, 2020) confirme que les programmes de formation des enseignants de FLE ne prévoient généralement pas de temps spécial à consacrer à l'étude des mécanisâmes nécessaires à l'enseignant, quand il est confronté à une situation d'évaluation des apprentissages dans la classe.

Pour cela, les programmes de formation doit préparer les futurs enseignants d'utiliser des échelles d'appréciation pour juger l'apprentissage de leurs étudiants, d'utiliser des critères et des indicateurs dans leur évaluation pour parvenir à des jugements équitables, et encourger les futurs enseignants à participer à la construction des critères d'évaluation pour qu'ils puissent gérer leurs propres apprentissages, de mettre en place des activités de sensibilisation adressées aux étudiants sur certains outils d'autoévaluations jugés nouveaux tells que le portefeuille.

On doit accroder une grande importance aux compétence professionnelles tels (la gestion de la classe, évaluer l'apprentissage des élèves, intégrer la 
TIC dans l'apprentissage, planifier un cours ) dans la formation pratique qui constitue un point d'ancrage essential dans le cheminement professionnel. Par l'apprivoisement de tous activités et tâches professionnelle au sein d'équipes éducatives au cours des stages leur assurent une formation professionnelle intégrée et soutenue et valorisante. Ce qui permet d'interrelation entre les dimensions théoriques et pratiques de l'exercice de la profession.

\section{3)La faiblesse au côté linguistique:}

Beaucoup d'études comme (El-Alawy,2015; Mohammed,2012; El-Fakharany, 2010; Hafez, 2009, AIADL, 2019; Ismail,2016)ont montré que le niveau des étudiants de la section de français en facultés de pédagogie est médiocre en ce qui concerne la compréhension orale. Ces études ont également recommandé de développer cette compétences auprès des étudiants des facultés de pédagogie en raison de son importance.

Etude de( Ismail, 2016) et Mohammed, 2015) a recommandé d'accorder un soin particulier à la compréhension orale en classe FLE en entrainant les enseignants et les futurs enseignants de FLE aux étapes d'enseignement de la compréhension orale et en faisant de la compréhension orale une partie des tests du français dans les écoles.

Les résultats d'autres recherches et études telles que (Parpette, 2008) préconisent que le développement des habiletés de la compréhension orale constituent la base essentielle pour développer les 
autres habiletés dans le domaine de la didactique de FLE. De tells résultats confirment ce que recherche l'étude en cours.

(Goullier, 2008 :56 ) met l'accent sur des faiblesses de l'enseignement des langues dans les points qui suivent :

1- La difficulté à se défaire de l'illusion du modèle du locuteur natif comme objectif implicite de l'enseignement dispensé à l'ensemble d'une classe d'âge.

L'absence de confiance d'un grand nombre d'élèves dans leurs aptitudes à progresser dans la maitrise des langues étudiées ; entraînement encore déséquilibré aux différentes activités langagières.

Le bref survol des éléments de la situation d'enseignement/ apprentissage de la langue française à la Faculté de Pédagogie de l'Université de Damiette nous a permis de mettre en relief certaines causes, qui selon nous, sont responsables de la faiblesse des compétences orales des futurs enseignants du FLE

Selon nous les causes de la faiblesse des compétences orales des futurs enseignants du FLE en Égypte en général et à la faculté de pédagogie de l'université de Damiette en particulier peuvent se résumer en :

1- L'absence d'une notion précise de ce que comporte la compétence orale et ses composantes. 
2- La difficulté à définir des objectifs et des activités spécifiques pour l'enseignement de la compétence orale.

3- L'absence des cours spéciaux de formation, même pendant les cours de phonétique où les séances du laboratoire de langue sont limitées à l'écoute et à la répétition des sons, des mots ou des phrases.

4- Lacune des matériels audio oral et visuel, etc. exercent un frein sur la possibilité de réaliser un enseignement à partir d'un support oral.

On peut dire le futur enseignant exige la pratique et la maîtrise des compétences didactiques précises convenables à sa nature, caractéristiques, et a ses objectifs d'enseignement telles que employer TIC dans et dehors la classe,

\section{Utilisation la plateforme dans . développement les compétences professionnelles:}

Il est remarquable que l'intégration et l'usage des technologies à des fins pédagogiques sont, à quelques exceptions près, peu présents dans la formation des futurs enseignants.

Comme l'estiment Mangenot et Louveau (2006), la meilleure manière d'exploiter les TIC (Technologies de l'information et de la communication) et plus particulièrement Internet est de la communication) et plus particulièrement Internet est de s'appuyer sur ces approches (par les tâches ou actionnelle) pour apprendre une langue . pour cela, une tâche est définie 
comme un agencement d'activités d'apprentissage (appellées par certains"micro-tâches" ou "soustâches", appuyé sur des ressources et prévoyant une production (Dejean\& Mangenot,2006,311-312).

les chercheurs comme(Othman Al-Qahtani ,2016) confirment que le développement professionnel est lié aux activités d'enseignant dans les processus d'enseignement et d'apprentissage afin de développer ses performances professionnellement et académiquement ou dans les dimensions culturelles générales et académiques et d'utiliser les innovations, les outils de communication et la technologie dans l'enseignement de la langue française, Les enseignants ayant considéré la plateforme pédagogique comme un présentiel enrichi, disent ainsi que la plateforme de cours en ligne fait généralement partie de leur milieu de vie.

La plateforme rend le processus d'évaluation plus efficace pour les enseignantes par la correction automatique de certains types de quiz. Les enseignants peuvent aussi publier des réponses aux questions que les etudiantes leur posent dans des groups de discussions. Cette formule réduit consiidérablement le temps passé à repondre individuellement a chaque étudiant

D'autant que ce sont souvent les mêmes questions qui reviennent au cours de la session. 
Les tâches de l'enseignant deviennent difficiles à mener du fait que son savoir professionnel ne lui permet plus de mettre en oeuvre des actions d'enseignement qui puissant développer de nouvelles compétences chez l'apprenant.ces compétences professionnelles aident tout enseignant à détecter pourquoi ses élèves ont des difficultés a apprendre, ainsi que comment render leur apprentissage plus motivant.

Dans ce contexte, la plateforme pédagogique est utilisée pour mettre en ligne des cours, des exercices et corrigés types, des questionnaires. Les enseignants justifient le peu d'investissement qu'ils ont concédé à l'utilisation de la plateforme par les tâches pédagogiques et les responsabilités qu'ils assument et, par voie de conséquence, le temps qu'ils auraient dû y consacrer pour la mise en ligne de contenus pédagogiques. Hormis un enseignant chercheur se présentant très actif et travaille sur le « $e$-Learning » où il utilise la plateforme pédagogique en lien avec ses travaux de recherches, l'usage de la plateforme comme lieu de stockage ou de dépôt est justifié par le fait de recourir à l'usage d'autres outils de communication en dehors de la plateforme d'apprentissage. Le rapport de certains enseignants à la plateforme pédagogique est un reflet contextualisé du rapport avec les supports de cours en général. Les cours en présentiel sont plus importants que les cours mis en ligne.( Ghaouthi, 2015:23)

La presente recherhce met en relation trois composantes : l'intégration de LMS, la formation via plateforme «Microsoft Teams » contribuent elles aussi 
de manière positive au développement des compétences professionnelles des enseignants en début de carrière.

\section{* ÉTUDE EXPÉRIMENTALE}

Cet axe vise à éxposer les étapes procédurales et les outils préparés par la chercheuse afin d'éxcuter la partie experimentale de la recherche. Cette démarche se représente à :

\section{Méthode de la recherche:}

La cherhceuse a utilisé une méthode de type expérimental en appliquant les outils de la recherche pour vérifier leurs impacts sur le développement des compétences professionnelles auprès les futurs enseignants. En outre, nous avons recouru à la méthode descriptive pour en développant le cadre théorique de la recher

\section{Échantillon de la recherche:}

Nous avons choisi trente étudiants de troisième année, section de français, à la faculté de pédagogie de Damiette, de l'année universitaire 2020/2021, comme groupe expérimentale. Nous avons choisi ces étudiants parce qu'ils s'entaînent Durant la troisième année aux compétence professionnelles et le stage pratique ils sont sur le point de démarrer l'enseignement du français langue étrangère. 
d'apprentissage :

Cette plateforme visé, en général, a sensibiliser le futur enseignant sur l'importance de la classe dans l'oeuvre éducative, a améliorer sa gestion dans son contexte actuel, à enricher les méthodes et techniques d'enseignement, a renforcer les compétences du futur enseignant. Et sa capacité d'évaluer ses élèves , intégrer la technologie dans la classe, et son planifier d'un cours.

L'expérimentation de la plateforme proposée a eu lieu du 17 fevrier 2021 au 12 avril 2021, Durant le deuxième semester de l'année universitaire 2020/2021 , à travers les tâches d'apprentissage déjà préparées. Elle a duré deux mois au rythme d'une tâche d'apprentissage par semaine pour bien s'entraîner aux compétences professionnelles,.

Les documents et les tâches d'apprentissage sont classés par ordre alphabétique sans une structuration rigoureuse, «Microsoft Teams» deviendrait vite impraticable. Ainsi, les étudiants qui n'ont pas pu assister au cours, peuvent aisément rattraper la ldu fait que tous les documents, y compris la vidéo du cours , sont disponibles à tout moment. Nous avons aussi utilisé la fonction forums pour que les étudiants puissent charger sur Microsoft Teams leurs dialogues écrits Ainsi , ils avaient plusieurs modèles de dialogues sur un même sujet pour approfondir leurs connaissances en les compétences professionnelles, Microsoft Teams nous a 
également permis de mettre sur la page d'acceuil des liens très utiles :dictionnaires, chaines de télevision françaises, sites français, etc. cette plateforme a surtout rendu possible la communication, grâce à son chat, entre les étudiants en ligne et l'enseignante. Dès qu'une faute de frappe s'est glissée, ce qui se produit fréquemment, nous intervenons pour rétablir la bonne orthographe du mot concerné.

\section{Expérimentation des outils de la recherche:}

Nous avons appliqué un pré-test à l'échantillon de la recherche le 12 février 2021. Ce pré-test a pour objectif premier d'évaluer les compétences professionnelles auprès les futurs enseignants . les résultats du pré-test ont indiqué la faiblesse du niveau des apprenants en ce qui concerne les compétences professionnelles en français.

Nous avons appliqué un posttest à l'échantillon de la recherche le 14 avril 2021 . ce posttest a pour objectif de vérifier le degré de progrès réalisé après avoir s'entrainé les tâches d'apprentissage et l'effet de la plateforme Microsoft Teams sur le développement les compétences professionnelles chez les participants. Nous avons ainsi fini notre expérience, qui a duré deux mois et deux jour, du 12 février 2021 au 14 avril 2021 


\section{RÉSULTATS DE LA RECHERCHE :}

Nous présentons les résultats obtenus selon les hypothèses de la recherche:

La recherche actuelle va essayer d ' examiner les hypothèses suivantes :

Pour vérifier la validité de la premiere hypothèse, qui stipule qu'il existe une différence significative au niveau <0.05 entre les moyennes des notes des étudiants au pré/posttest des compétences professionnelles en faveur du posttest", nous avons calculé d'abord la moyenne arithmétique des notes des étudiants au pré/posttest des compétences professionnelles en utilisant le tableau Excel. Ensuite, nous ayons appliqué le test $\mathrm{T}$ pour échantillons appariés afin de comparer les moyennes des résultats obtenus par les participants au pré/posttest des compétences professionnelles. Nous avons obtenu les résultats suivants :

\section{Tableau (1)}

Résultats du groupe expérimental à la pré- post post test en ce qui concerne l'application du test des compétences professionnelles;moyennes

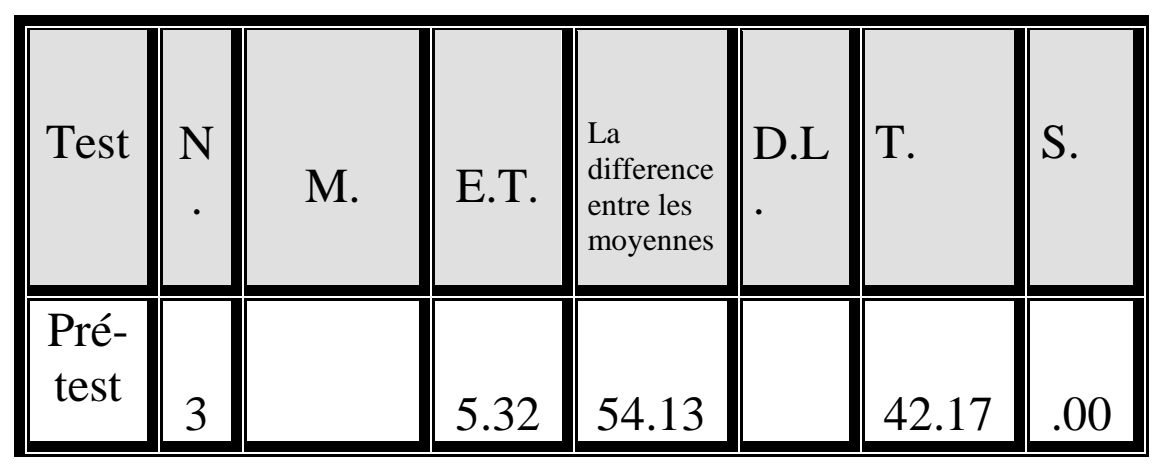




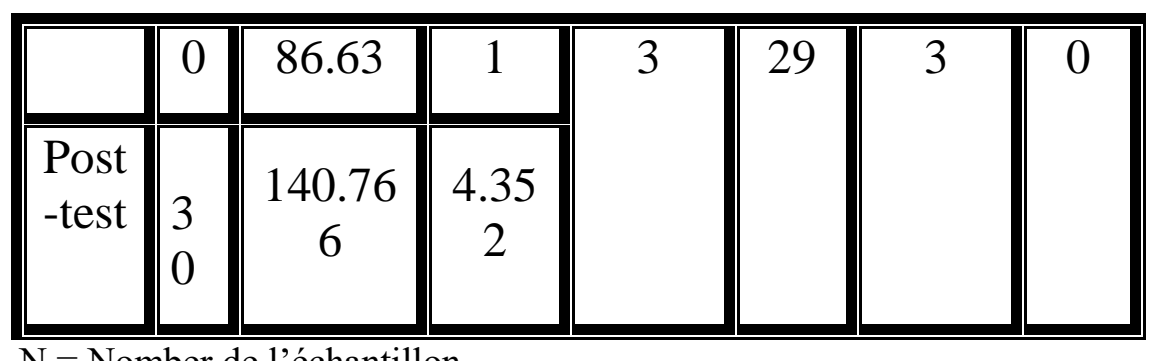

$\mathrm{N}=$ Nomber de l'échantillon.

M=Moyenne Arithméthique.

E.T=Ecart-Type .

$\mathrm{T}=$ Valeur de $\mathrm{t}$.

DDL=degree of liberte ( df ) degré de liberté (ddl)

$\mathrm{S}=$ Valeur Significative.

N.S = Valeur non Significative.

\section{Commentaire :}

Généralement ,en examinant le tableau (1), nous remarquons que la valeur de $\mathbf{T}$. qui concerne le pré-post application du test de la performance professionnelles ( en totale ) est(42.173), il est statistiquement significatives au niveau de (0,001). Ce qui met en valeur la progression dû à l'utilisation de notre programme proposé basé sur la plateforme électronique . Donc, on peut accepter la première hypothèse .

Le tableau suivant montre la valeur de " Carré d'éta", la valeur de "d" qui la rencontre et la taille d'effet envue de développer les compétences professionnelles auprès les futurs enseignants. 
Pour vérifier la validité de la deuxième hypothèse, qui stipule qu"'la plateforme électronique a une taille d'effet sur le développement des compétences professionnelles en FLE pour l'échantillon de la recherche" nous avons calculi l'eta-carre $\eta^{2}$ qui indique la taille d'effet (effect size) avec l'équation et le tableau 2 suivants:

$$
\begin{aligned}
& \boldsymbol{\eta}^{2}=\frac{T^{2}}{\boldsymbol{T}^{2}+\boldsymbol{D L}} \\
& \boldsymbol{\eta}^{2}=\text { Carré d’Éta } \\
& \boldsymbol{T}^{2}=\text { Le carré de la valeur “ } \boldsymbol{T} \\
& \mathbf{D L}=\text { le dégrée de liberté } \\
& \mathbf{N}=\text { Nombre des apprenants } \\
& 1778.5 \\
&
\end{aligned}
$$

$1778.5+29$

Tableau (2) valeur de l'eta-carré

\begin{tabular}{|c|c|c|c|c|c|c|}
\hline $\begin{array}{c}\text { Variable } \\
\text { indépend } \\
\text { ante }\end{array}$ & $\begin{array}{c}\text { Variable } \\
\text { dépendante }\end{array}$ & $\mathbf{T}$. & $\mathbf{T}^{2}$ & $\mathbf{\eta} \mathbf{2}$ & $\mathbf{d}$ & $\mathbf{V . L}$ \\
\hline \hline $\begin{array}{c}\mathrm{La} \\
\text { plateforme } \\
\text { proposee }\end{array}$ & $\begin{array}{c}\text { Les } \\
\text { compétence } \\
\text { s } \\
\text { professionn }\end{array}$ & 42.1 & 1778. & 0.9 & 15.6 & gra \\
\hline
\end{tabular}




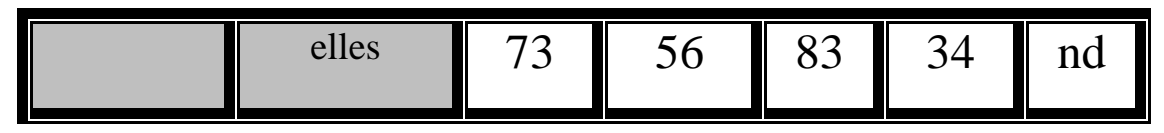

Le tableau 2 ci-dessus montre que la valeur de l'eta -carré $\eta 2$ est $(\mathbf{0 . 9 8 3})$ pour les compétences professionnelles. Cette valeur est supérieure à 0.8 . cela signifie que la plateforme électronique a un effet significatif sur le développement des compétences professionnelles en français pour l'échantillon de la recherche. Donc, notre deuxième hypothèse est aussi confirmée. En somme, nous pouvons dire que la plateforme électronique a pu atteindre les objectifs désirés du développement des compétences professionnelles pour notre échantillon.

En outre, l'équation suivante montre la relation entre la taille d'effet et le Carré d'Éta. (Mahssoub Abdel Kader,2006 :52)

$$
\mathrm{V}=\sqrt{\text { Carré d'Éta } \div(1-(\text { Carré d'Éta })}
$$

de Reda Moussad(2003) indique que:

Si la valeur de "d" $=\mathbf{0 . 2}$, c'est-à-dire que la taille d'effet est faible.

Si la valeur de "d" $=\mathbf{0 . 5}$, c'est-à-dire que $\div$ la taille d'effet est moyennes.

Si la valeur de "d" $=\mathbf{0 . 8}$, c'est-à-dire que la taille d'effet est grande.

En appliquant cette formule, on obtient les résultats suivants 


\section{tableau (3)}

la valeur d'éfficacite du programme

\begin{tabular}{|l||c|c||l|}
\hline & $\boldsymbol{\eta} \mathbf{2}$ & $\begin{array}{l}\text { Valeur } \\
\text { d'efficaci } \\
\text { té }\end{array}$ & $\begin{array}{l}\text { Taille } \\
\text { d'effe } \\
\mathbf{t}\end{array}$ \\
\hline $\begin{array}{l}\text { Test de la performance } \\
\text { professionnelle }\end{array}$ & $\begin{array}{l}0.983 \\
9\end{array}$ & 11.075 & $\begin{array}{l}\text { Grand } \\
\text { e }\end{array}$ \\
\hline
\end{tabular}

Commentaire général : les résultats de cette recherche s'accordent avec les résultats des études qui assurent à efficacité des « plateformes » comme l'étude d'Al Adl (2019), l'étude de El-Seify (2019), l'étude de Abdalla (2020), l'étude de Hafez (2009), l'étude Ghaouti (2015). (2008), l'étude de Hoda Elgamdi (2019), l'étude de Salem (2013), l'étude d'Elham elnaser(2013), l'étude de Omar (2014) l'étude de Bekhti(2011)De même notre recherche confirme le potentiel pédagogique et éducatif des plateformes électroniques pour l'enseignement / apprentissage d'une langue étrangère.

\section{Recommendations de la recherche:}

D'après les résultats de la recherche présentés, on peut présenter les recommandations suivantes:

- Adopter les standards de la qualité dans les programmes de formation l'enseignant offerts par la faculté de pédagogie;

- S'intéresser à l'entraînement individuel et collectif aux compétences nécessaires pour le processus de l'enseignement et les situations d'apprentissage; 
- Développer les programmes de formation (initiale et continue) des futurs-enseignants à la lueur des tendances modernes de l'enseignement;

\section{Suggestions de la recherche:}

En fait, la chercheuse croie que cette étude offrirait de nouveaux horizons aux chercheurs pour effectuer des études ayant pour but de développer d'autres compétences langagières en français a travers l'emploi des plateformes électroniques.

- Etude des effects des plateformes sur le développement de la compétence grammaticale chez les apprenants de FLE;

- Etude des effets des plateformes sur le développement de la compétence lexicale chez les apprenants de FLE;

- Etude des relations entre l'usage des plateformes et l'autonomie des apprenants de FLE; 


\section{Bibliographie :}

\section{1 ) Réfèrences en langue française :}

Abbas, M.( 2019). UnitéProposée Basée sur L'approche Actionnelle pour Le Developpement de Quelques Competences ProfessionnellesChez Les Futurs Enseignants de la Langue Francaise, Magasine des sciences éducatives, $\mathrm{No}(38)$, pp 54-84

Abdalla, O.(2020). Efficacité des Plateformes

D'apprentissage Électronique pour Développer des

Compétences de la Compréhension Orale en Français chez les Étudiants du Cycle Secondaire, Research in Teaching Languages, $\mathrm{VO}(1), \mathrm{NO}(10)$.

Ahmed, R. (2018) . "La formation professionnelle des futures enseignants de la section de français à la lueur de l'apprentissage collaborative en ligne", Département du curriculum et des methods d'enseignement, Thèse de doctorat, Université d'Assiut.

Al Adl, W. (2019). Plateforme Numerique D'apprentissage Pour Développer Les Compétences de Communication Orale en Fle, Magazine des sciences pédagogiques, No (39), pp1-22 .

Association américaine de l'apprentissage et du développement (2012). Connecting Research to Performance .ATD Research (Benoit, On dirait que"Connecting Research to Performance"regroupe plusieurs recherches. 
Est-ce vraiment le nom d'une recherche en particulier?)

Bekhti, B.(2011). Le Choix d'une plateforme Elearning et leur Rôle dans l'apprentissage et l'évaluation, Magasine des Sciences Humanitaire et

Sociale, $\operatorname{No}(6)$, pp54-73

Brunel, S ;Girard, P; Lamago, M . (2015).Des plateformes pour enseigner à distance : vers une modélisation générale de leurs fonctions. , France.

hal01128532 disponible surhttps://hal.archivesouvertes.fr/hal-01128532

Mangenot, F. et Louveau, E. (2006). Internet, tâches et vie réelle. Le français dans le Monde, 45,82-90.

Dejean, C. \& Mangenot, F. (2006). Tâches et scenario de communication dans les classes virtuelles, les Cahiers de l'Asdifle 17,310-321.

Develay, M.(1994). De la coupe aux lèvres. Dossier sur l'enseignement stratégique. Dimensions, septembre ,6-7.

Dumouchel, G.(2016).” Les compétences informationnelles des futurs enseignants québecois sur le Web ", (thèse de doctorat). Université de Montréal. 
Ibrahim, M.(2020). Utilisation de l'approche réflexive pour modifier les croyances des enseignants du FLE concernant l'évaluation des apprentissages, Research in Teaching Languages, $\operatorname{Vol}(1), \operatorname{No}(10)$.

Gaafar, A. (2015). “ Efficacité d'un programme d'apprentissage par proposé basé sur l'approche problèmes pour le développement de quelques compétences en gestion de classe chez les enseignants

débutants de la langue française", thèse de Doctorat, Département de Curricula et de Méthodologie, Faculté de pédagogie, Université d'Ain Chams.

Ghaouti, Z.(2015). Pratiques de la plateforme de Cours en ligne par les Enseignants Universitaire: l'émergence de Nouvelles Pratiques de Communication par les Etudiants", Magazine des sciences humanitaire

et sociale, No.(19), pp21-28,

Hafez,H.(2009). Efficacite de l'approche d'apprentissage par problèmes pour le développement de quelques competences professionnelles chez les futurs enseignants de la faculté de Pédagogie, association egyptienne de curriculum et méthodologie, faculté de pédagogie, université de Ain Chams.NO(144).

Hamdan,D.(2019). Les compétences Informationnelles

Necessaires A la Formation 
Contenue Et le Degre de leur Presence Chez les Enseignants Actuels Du Français langue Etrangere, la

Magasine Pédagogique, Vol(65), PP1-44

Ecoutin, E.\& Even, N.( 2001). Mise en œuvre des plates-formes de formation à distance. Les documents. Fiche pratique $\mathrm{n}^{\circ} 3$, disponible sur http://www.algora.org (consulté en novembre 2021).

Elfakharany, Ch .(2017). "Développement des compétences linguistiques et communicatives des futurs-enseignants de français langue étrangère de

la faculté de pédagogie à la lueur de l'approche interculturelle via Internet", thèse de doctorat, faculté de pédagogie, Université de Tanta.

El seify ,M . (2019): Utilisation de la Plateforme MOODLE dansl'acquisitiondes perspectives de l'enseignement et son effet sur le développement des competences professionnelles auprès des étudiants de diplôme general à la faculté de pédagogie, Magasine

Faculté de pédagogie, Vol (34), NO(3), pp98156.

Ezz El-arab, M. (2019). Utilisation de la Stratégie de l'apprentissage Situationnel pour développer quelques compétences professionnelles auprès les étudiants du Diplôme général du Françaisà la Faculté de pédagogie, Magasine de faculté de pédagogie, vo. 34,No.3,pp297 
Le Boterf, G. (2004). Construire les compétences individuelles et collectives, édition Eyrolles,

Mangenot F. (2002) .« Chapitre 6 Analyse sémiopragmatique des forums

pédagogiques sur Internet », disponible sur http://w3.udefis.pdf

grenoble3.fr/espace_pédagogique/chap6-

Moftah, S. (2016).La professionnalisation des enseignants du FLE : Quelle(s) compétence(s) professionnelle(s) ? Quelle évaluation?",

Université de Taëz, Magazine de faculté de langues, No.13, pp119-142, Yémen,

Osman, G.(2018). “Programme Basé sur l'autoApprentissage Pour Développer

Quelques Compétences de l'évaluation des Apprentissages Chez les Enseignants du FLE”, Faculté de pédagogie, Université de Minia,

Omar, H.(2014). " Efficacite d'un programme propose en utilisant le e-learning sur le développement des compétences de la communication orale en français chez les étudiants de la section de français de pédagogie", Thèse de doctorat, faculté de pédagogie, Université d'Al-Azhar. 
Parpette Ch., (2008). De la compréhension Orale en Classe à la Réception Orale en Situation Naturelle: une Relation à interroger, l'Alsace au Coeur du plurilinguisme, Recherches en didactiques des langues, Les Cahiers de 1'Acedle, Vol.5,No.1 Radi , S.(2001). Les sciences en Egypte. Ministère des Affaires Etrangères \&), la Commission européenne. France. Paris.IRD.

Salem, H.(2013). Une cyberenquête proposée pour développer les compétences de l'expose orale chez les étudiants de FLE à la faculté de pédagogie de Sohag, Journal of Arabic Studies in Education\& Psychology,NO(44), PART(3), PP1-62.

Seyam, W(2017). Développer Pour Authentique evaluation'L Chez Professionnelles Competences Certaines de Faculte La a Enseignants Futurs les Pédagogie, faculté de pédagogie,

Université de Aim Shams, Magazine de lecture et écriture, No 228, pp78-124, Available sur 875664/Record/com.mandumah.search://http

Talbot, L. (2007). Quelles compétences professionnelles pour les

professeurs des écolesece. In M. Bru, \& L. Talbot (dir.), Des competences pour enseigner : entre objets sociaux et objets de

recherche, Rennes, Presses universitaires de Rennes. 
Tartif, J.(2006). L'évaluation des compétences Documenter le parcours de

Éducation.

développement. Montréal: Chenelière

Paquay, Léopold (1994). «Vers un référentiel des competences professionnelles de l'enseignant ? », in Les professions de l'éducation, ${ }^{\circ} 16$, Paris, INRP, coll. Recherche et formation, 7- 38.

Proulx , S., Sint-Charles, J.(2004): l'appropriation personnelle d'une innovation:le cas d'internet l'importance des réseaux d'appui", informations sociales, 116, pp80-89.

Peraya D., Viens J. (2005) . «Relire les projets « TIC et innovation pédagogique»: Y-a-t-il un pilote à bord, après Dieu bien sûr... ", disponiblesurhttp://tecfa.unige.ch/ peraya/homepage/p ubli/05_TICE_et_innovations $\% 20 \_p$

\%E9dagogogiques.pdf (consulté le 28/11/2014)

Proulx S. (2001). «Usages de l'Internet: la «penséeréseaux» et l'appropriation d'une culture numérique », disponible sur sergeproulx.uqam.ca/wpcontent/.../2001- proulx-usages-de-1-in-69.pdf

Komis,V., Depover, C., Karsenti, T., Tselios, N.\& Filippidi, A. (2013). Comprendre l'usage des plateformes d'enseignement et les outils Wed 2.0 dans des contexts universitaires de formation hybride: aspects méthodologiqes. Formation et profession, 21(2), 52-69. 


\section{Références en langue Anglaise:}

Abd El-Gawaad, R. (2016)." The Effectiveness of

Virtual Learning Environment in Developing

Teaching Efficiency of Basic Education Student

Teachers of English", Aresearch submitted in fulfillment of the requirements for PH.D. dissertation in curriculum and English language teaching, Faculty of Education, Assuit University.

Dohn, N.B.(2009). Web 2.o:internet tensions and evident challenges for education. International Journal of Computer- supported Collaborative Learning, 4(3),343-363.

Gowda, A.(2018). "The Effectiveness of Using a Grammer Program Based on Virtual Learning (VLE) on Developing the Writing Skill for Third Year in Prep Stage", Thesis of master's Degree in Education,

Curriculum \& Instruction Department, Faculty of Education, Beni- suef University. 


\section{Références en langue Arabe:}

إلهام الناصر .(2013). الإدمودو تصور جديد للتعليم و التنريب ـ مجلة التدريب و التقنية.(172). الإدود تصور جناح من

http://altadreeb.net/articleDetails.php?id=942\& issue $\mathrm{No}=32$

حسن شحاته , زينب النجار (2003). معجم المصطلحات التربوية والنفسية, القاهرة, الدار الدصرية اللبنانية رضا مسعد.(2003). " حجم الأثر(:أساليب إحصائية لحساب الأهمية

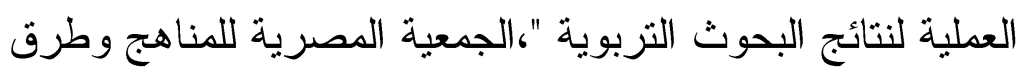

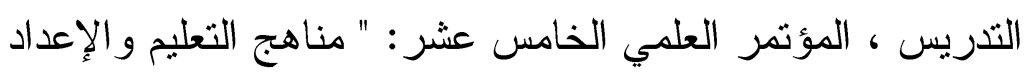
للحياة المعاصرة ، جامعة عين شمس ، 21-22-يونيو ، صص: لكصن . 1:32

محمد عطية خميس(2003) ـ منتوجات تكنولوجيا التعليم, القاهرة, دار الكلمة

نادر أحمد بيومى(2018) :"تطوير بيئة تعلم افتراضية قائمة على القى الامج بين أدو ات نظم إدارة التعلم الإلكترونى وبيئات التعلم الثخصية لتتمية مهار ات التصميم التعليمى لطلا تكنولوجيا التعليم بكلية التربية", رسالة دكتور اة, قسم تكنولوجيا التعليم, كلية التربية, جامعة دمياط. هدى سعد الغامدى(2019). فاعلية استخدام منصة تعليمية فى تتمية

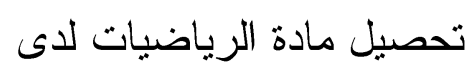




\section{مجلة كلية التربية - جامعة دمياط العدد 79 أكتوبر 2021}

طالبات الصف السادس الإبتدائى, المجلة التربوية لتعليم,

الكبار ,المجلد الأول, العدد الثانى, كلية التربية , جامعة

أسيوط 